Tecno Lógicas

ISSN 0123-7799

Vol. 38, No. 20, pp. 27-39

Enero-junio de 2017

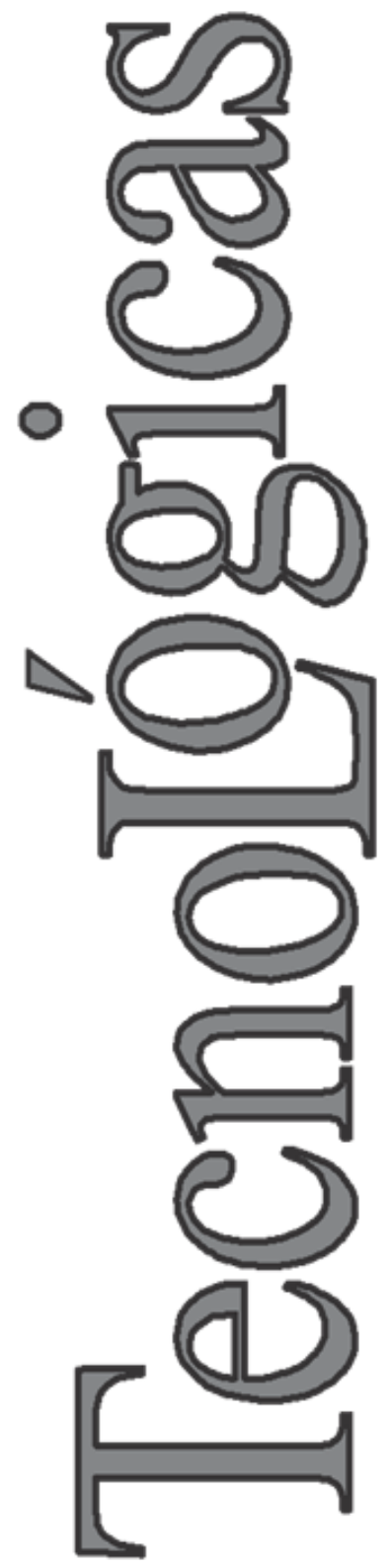

(C) Copyright 2015 por autores y Tecno Lógicas Este trabajo está licenciado bajo una Licencia Internacional Creative Commons Atribución (CC BY)

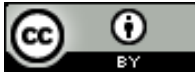

\section{Diseño de relajadores de campo eléctrico usando optimización por enjambre de partículas y el método de elementos finitos}

\section{Electric field relaxing electrodes design using particle swarm optimization and finite elements method}

Jhon E. González-Pérez y Diego F. García-Gómez²

Recibido: 09 de junio de 2016, Aceptado: 05 de diciembre de 2016

Cómo citar / How to cite

J. E. González-Pérez y D. F. García-Gómez, "Diseño de relajadores de campo eléctrico usando optimización por enjambre de partículas y el método de elementos finitos", Tecno Lógicas, vol. 38, no. 20, pp. 27-39, 2017.

1 Ingeniero Electricista, Escuela de Ingeniería Eléctrica y Electrónica, Facultad de Ingeniería, Universidad del Valle, Florida-Colombia, jhon.e.gonzalez@correounivalle.edu.co

2 PhD. en Ingeniería Eléctrica, Electrónica y Automática, MSc. en Sistemas de Generación de Energía, Ingeniero Electricista, Escuela de Ingeniería Eléctrica y Electrónica, Grupo de Investigación en Alta Tensión (GRALTA), Facultad de Ingeniería, Universidad del Valle, Cali-Colombia, diego.garcia@correounivalle.edu.co 


\section{Resumen}

En este artículo se presenta una metodología para el diseño de electrodos relajadores de campo eléctrico, la cual se basa en un proceso de optimización desarrollado mediante la técnica metaheurística de optimización por enjambre de partículas. La función objetivo del proceso de optimización incluye el modelo electro-estático del equipo de alta tensión, el cual es resuelto mediante el método de elementos finitos. La metodología propuesta fue implementada por medio de las herramientas computacionales COMSOL Multiphysics y MATLAB, y validada a través del diseño de los electrodos relajadores de campo eléctrico de un divisor de tensión resistivo para medición de ondas de impulso de tipo rayo.

\section{Palabras clave}

Optimización por enjambre de partículas, Método de elementos finitos, Diseño de equipos de alta tensión, Electrodos relajadores de campo eléctrico.

\section{Abstract}

In this paper, a methodology for design of electrical field relaxing electrodes is shown. This design methodology is based in an optimization process carried out by particle swarm optimization technique. The objective function of the optimization process, include the electro statics model of the high voltage equipment that is solved by the finite element method. The proposed methodology was implemented using the computational tools Matlab and Comsol. This methodology was validated by designing the electric fields relaxing electrodes in a high voltage resistive divider, which used in measurement of lightning impulse waves.

\section{Keywords}

Particle swarm optimization, Finite element method, High-voltage equipment design, Electric field relaxing electrodes. 


\section{INTRODUCCIÓN}

Los equipos de alta tensión, como es el caso de los divisores de tensión, usualmente tienen una disposición física vertical, la distribución de la tensión entre el terminal de alta tensión y las conexiones a tierra no es uniforme debido a la presencia de capacitancias parásitas [1]. Cuando el campo eléctrico local supera un umbral crítico, se presentan descargas, lo cual puede resultar en la degradación del aislamiento del equipo o aumento de la temperatura del mismo. La distribución no uniforme de la tensión también puede crear grandes esfuerzos eléctricos en los elementos que constituyen el equipo de alta tensión. La solución a estos problemas es la implementación de dispositivos externos metálicos, denominados genéricamente como electrodos relajadores de campo eléctrico (ERCE), cuyo fin es redistribuir la tensión y reducir el valor máximo del campo eléctrico en los elementos próximos a la entrada de tensión.

Los electrodos relajadores de campo eléctrico, en algunos casos también denominados anillos relajadores de campo eléctrico, son dispositivos externos de material conductor, generalmente en forma de toroide, que se encuentran ubicados en las inmediaciones de los terminales de los dispositivos de alta tensión. La principal función de los electrodos relajadores de campo eléctrico es distribuir y homogenizar la intensidad del campo eléctrico en el equipo de alta tensión y así: (i) reducir las causas de deterioro y (ii) disminuir los valores máximos de intensidad de campo eléctrico en los elementos superiores, tales como condensadores o resistencias, para que estén por debajo del umbral de descargas corona, previniendo así su aparición.

Los electrodos relajadores de campo eléctrico se usan habitualmente en los generadores de Van de Graaff, aisladores eléctricos, pararrayos y otros equipos de alta tensión. Existen diferentes tipos de electrodos relajadores de campo eléctrico, cada uno de estos permite blindarse contra el efecto corona. En el trabajo reportado por Pattanadech et al [2] se muestran algunos tipos de ERCE.

Generalmente el diseño de los electrodos relajadores de campo eléctrico, implica encontrar los valores adecuados de los parámetros geométricos que permiten lograr una distribución lineal del voltaje en el equipo de alta tensión. Esta tarea es compleja de llevar a cabo de forma experimental, puesto que es difícil hacer mediciones sin que sean afectadas por campos eléctricos circundantes. Sin embargo, en los últimos años se han adelantado investigaciones relacionados con el análisis mediante el método de elementos finitos (MEF) para ayudar a la solución del comportamiento eléctrico de equipos de alta tensión.

El diseño de los ERCE, consiste en buscar las dimensiones geométricas que produzcan una adecuada distribución de tensión en el equipo de alta tensión; por lo tanto, puede abordarse como un problema de optimización. En este sentido la técnica metaheurística de inteligencia artificial, denominada optimización por enjambre de partículas o PSO por sus siglas en inglés (Particle Swarm Optimization), es una herramienta útil para este propósito pues explorara de manera más eficiente el espacio de búsqueda, respecto de otras técnicas similares [3]. Adicionalmente, las técnicas de optimización como PSO tienen la capacidad de trabajar con funciones objetivo que no requieren ser funciones matemáticas derivables, por tanto, permite usar modelos resueltos a través del método de los elementos finitos.

En diferentes trabajos se muestra el empleo de las técnicas MEF y PSO en la determinación de los parámetros de diseño de componentes de equipos eléctricos de alta tensión, como por ejemplo en descargadores de sobretensión [4] y aisladores [5]-[7]. Existen otras aplicaciones donde también se emplean estas dos técnicas para el diseño; por ejemplo, en el diseño de motores de DC [8], en la optimización del 
diseño de sensores electroestáticos [9] y en el diseño de parachoques de automóviles [10].

El diseño optimizado de los ERCE es una tarea compleja que puede abordarse como un problema de optimización, en el cual un modelo de elementos finitos del equipo de alta tensión, incluyendo su ERCE, son el núcleo de la función objetivo.

En este artículo se muestra la implementación de una metodología de diseño de electrodos relajadores de campo eléctrico (ERCE) para equipos de alta tensión, mediante la ayuda de las técnicas de PSO y MEF. La metodología propuesta en el presente trabajo mejora el diseño de los ERCE, disminuyendo así el riesgo de que los elementos constitutivos del equipo de alta tensión sean afectados por excesivos esfuerzos eléctricos y por ende garantizando su vida útil.

\section{METOdOLOGíA}

La técnica PSO fue propuesta por primera vez en el año 1995 por Eberhart y Kennedy [11]. Esta técnica es basada en el comportamiento social de grandes grupos de animales denominado algoritmo de nubes (también enjambre o cúmulos) de partículas que más tarde se llamó optimización por enjambre de partículas (Particle Swarm Optimization, PSO).

En la técnica PSO, una serie de organismos sencillos (partículas) que se sitúan en el espacio de búsqueda de algún problema, y evalúan la función objetivo en su ubicación actual. Cada partícula determina su movimiento a través del espacio de búsqueda mediante la combinación de algunos aspectos de su propia y mejor ubicación (mejor aptitud) comparadas con uno o más miembros del enjambre, con algunas perturbaciones aleatorias. Después de cumplir con las condiciones iniciales, todas las partículas se mueven y así sucesivamente se repite hasta que finalice el algoritmo [12], [13]. Los algoritmos basados en nubes de partículas se emplean en diferentes campos de investigación para la optimización de problemas complejos. El algoritmo es una técnica metaheurística en concreto [14].

El diseño adecuado de los ERCE implica encontrar aquella geometría que permite obtener una distribución lineal del voltaje a lo largo del equipo de alta tensión, para ello se emplea un modelo electrostático que se resuelve mediante elementos finitos. En este modelo la geometría de los ERCE es de tipo parametrizada, es decir, sus dimensiones se pueden considerar como variables independientes cuyos valores son suministrados de forma externa al modelo MEF por el algoritmo de optimización, el cuál es basado en la técnica PSO. En la 0 se presenta el diagrama de flujo general de la metodología propuesta para el diseño de los ERCE, empleando el método de elementos finitos y las técnica de optimización metaheurística PSO.

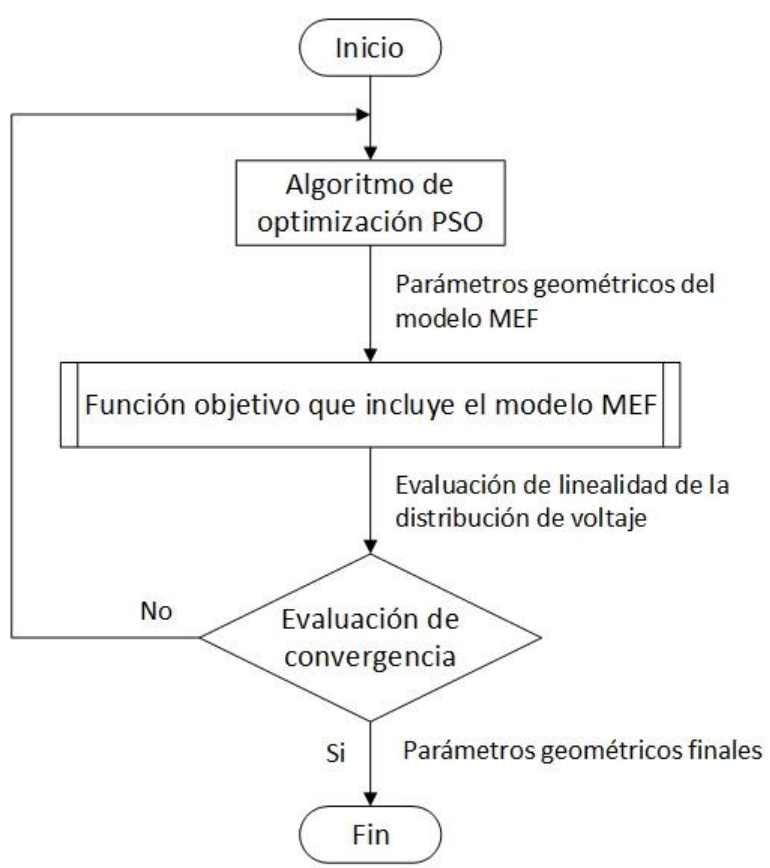

Fig. 1. Diagrama de flujo general de la metodología propuesta. Fuente: Autores.

La metodología inicia definiendo las variables geométricas de los electrodos relajadores de campo eléctrico (ERCE), que son los parámetros que se desean optimizar 
mediante la técnica PSO. En esta parte del proceso es conveniente definir qué tipo de algoritmo PSO emplear, sus parámetros específicos, el rango de las variables (espacio de búsqueda), el número de partículas y el número máximo de iteraciones, entre otros.

Un aspecto importante en el algoritmo PSO es fijar qué criterio de parada o de convergencia se debe de usar, ya que de ello dependerá en gran medida la relación entre la calidad de los resultados y el tiempo empleado en la optimización. Como criterio de convergencia se puede usar un umbral (threshold), que establece un nivel de error aceptable entre el resultado ideal y el óptimo obtenido de la función objetivo. $\mathrm{Al}$ escoger un adecuado valor de umbral, se asegura que los tiempos de búsqueda se reduzcan; sin embargo, es posible que, al detenerse el proceso de búsqueda, se estén descartando soluciones mejores a la ya obtenida. También puede emplearse un criterio de parada consistente en dejar que el proceso de optimización agote el número máximo de iteraciones. De esta forma se asegura encontrar la mejor de las soluciones posibles, pero el tiempo empleado en encontrarla posiblemente sea mayor que el empleado con un criterio de convergencia de tipo umbral.

En cada iteración, los parámetros geométricos de los ERCE, provenientes del algoritmo PSO, se introducen a la función objetivo para complementar el modelo de elementos finitos. A través de la función objetivo se obtiene la distribución de tensión a lo largo del equipo y entonces se cuantifica su linealidad. Por último, a partir del resultado de la función objetivo, se evalúa la convergencia o parada del proceso de optimización. En caso de que el criterio de parada se cumpla, estos valores de los parámetros geométricos serán los correspondientes al diseño final del ERCE; en caso contrario, el algoritmo PSO genera un nuevo conjunto de parámetros geométricos y el proceso se repite.

\subsection{Algoritmo PSO}

En PSO los agentes de búsqueda denominados partículas, intercambian información para mejorar el proceso de exploración en el espacio de búsqueda. La ubicación de los agentes del enjambre se inicia de forma aleatoria y cada posición individual evoluciona iteración tras iteración, modificando su dirección en función de las direcciones de las partículas de su vecindario. Para ello PSO almacena la experiencia propia o historia de cada partícula y entonces cada partícula del enjambre decide su nueva dirección en función de la mejor posición por la que pasó anteriormente y de la mejor posición que ha encontrado cualquier otra partícula del enjambre. En la metodología propuesta, las partículas contienen información de los parámetros geométricos de los ERCE.

La trayectoria de las partículas dentro del espacio de búsqueda está definida con base en el vector de ubicación $\vec{x}$ y el de velocidades $\vec{v}$, los cuales son sumados para obtener la nueva dirección. Justamente estos cambios de trayectoria son los que determinan la característica principal del algoritmo PSO, ya que a través de los mismos las partículas son forzadas a buscar soluciones en las áreas más promisorias del espacio de búsqueda. Cabe destacar que si los valores de $\vec{v}$ no se modificarán, la partícula sólo se movería con pasos uniformes en una única dirección.

$\mathrm{Si}$ se considera un problema de $\mathrm{N}$ dimensiones, donde las dimensiones representan el número de parámetros geométricos asociados a los ERCE del equipo de alta tensión, para la i-esima partícula de $n$ dimensiones, con $n \in[1-N]$, la evolución del algoritmo en su forma básica está determinada por 0 y 0 [13].

$$
\begin{gathered}
v_{i}(t+1)=v_{i}(t)+c_{1} * r_{i 1} *\left(x_{i}^{\text {pbest }}-x_{i}(t)\right) \\
+c_{2} * r_{i 2} *\left(x_{j}^{\text {gbest }}-x_{i}(t)\right)
\end{gathered}
$$


Donde $v_{i}(t+1)$ es el valor de la velocidad de la i-esima partícula en el instante $(t+1), \quad v_{i}(t)$ es la velocidad precedente, $c_{1}$ es el coeficiente de aprendizaje cognitivo, $c_{2}$ es el coeficiente de aprendizaje social, $r_{i 1}$ y $r_{i 2}$ son los valores aleatorios uniformemente distribuidos en el rango $[-1,1], x_{i}(t)$ es la posición actual de la partícula en la dimensión $n, x_{i}^{\text {pbest }}$ es la posición de la partícula $i$ con la mejor solución encontrada, y $x_{j}^{\text {gbest }}$ es la posición de la partícula del enjambre que encontró la mejor solución hasta el momento. En la ecuación anterior pbest representa el mejor valor de la función objetivo encontrado por cada i-esima partícula, mientras que gbest representa el mejor valor de la función objetivo obtenido hasta el momento por cualquiera de las partículas del enjambre.

Algunas de las variaciones del algoritmo son PSO con factor de inercia en el cual se agrega un término adicional $(w)$ que multiplica a la velocidad precedente y PSO con factor de constricción, que consiste en multiplicar 0 por un factor $\chi$. Ambas variantes se han desarrollado para ayudar al enjambre de partículas a ser más eficiente en el proceso de exploración.

La influencia relativa de la memoria que lleva asociada cada partícula (pbest) es denominada influencia cognitiva, mientras que la memoria del mejor valor encontrado por alguna partícula de la población (gbest) es denominada influencia social. Ambas influencias determinan la velocidad de aprendizaje del enjambre y esto permite determinar con qué rapidez el enjambre convergerá a la solución [15].

\subsection{Función objetivo (Fo) y evaluación de convergencia}

El núcleo de la función objetivo es el modelo electroestático de elementos finitos del equipo de alta tensión, incluyendo su ERCE. En este modelo previamente deben definirse la geometría del equipo de alta tensión, las propiedades de los materiales involucrados (en este caso las permisividades eléctricas relativas), las condiciones de contorno o de frontera y las tensiones de entrada. La geometría del ERCE se construye de forma parametrizada, de tal manera que esta quede definida con los valores provenientes del proceso de optimización mediante PSO. En la 0 se presenta el diagrama esquemático de la función objetivo.

Una vez se introducen los parámetros geométricos del ERCE provenientes del algoritmo PSO, la geometría del modelo MEF queda totalmente definida y entonces se puede comenzar el proceso de discretización o generación de la malla, para finalmente proceder a la formulación y solución de las ecuaciones diferenciales parciales (PDE), asociadas al modelo electrostático.

Las herramientas MEF basan la solución del modelo de campos eléctricos estáticos utilizando el potencial eléctrico $\boldsymbol{V}$ al representar la ley de Gauss como la ecuación de Poisson (3).

$-\nabla\left(\varepsilon_{0} \nabla V-P=\rho\right)$

Donde $\boldsymbol{E}$ corresponde a la intensidad de campo eléctrico, $\boldsymbol{D}$ es el desplazamiento eléctrico o densidad de flujo eléctrico, $\boldsymbol{\varepsilon}_{\mathbf{0}}$ es la permitividad del vacío, $\boldsymbol{P}$ es el vector de polarización eléctrica y $\boldsymbol{\rho}$ es la densidad de carga eléctrica.

Después de obtenida la solución del modelo MEF, se define el contorno o trayectoria de interés en la que se desea conocer la distribución del campo eléctrico. Como información complementaria, también se pueden obtener los esfuerzos eléctricos sobre esta trayectoria.

Obtenida la solución de la distribución del voltaje en el equipo de alta tensión, se determina su grado de linealidad por medio de una regresión lineal, que implica encontrar cual es el conjunto de datos que mejor se ajuste a una línea recta. Esa evaluación de la linealidad del voltaje se efec- 


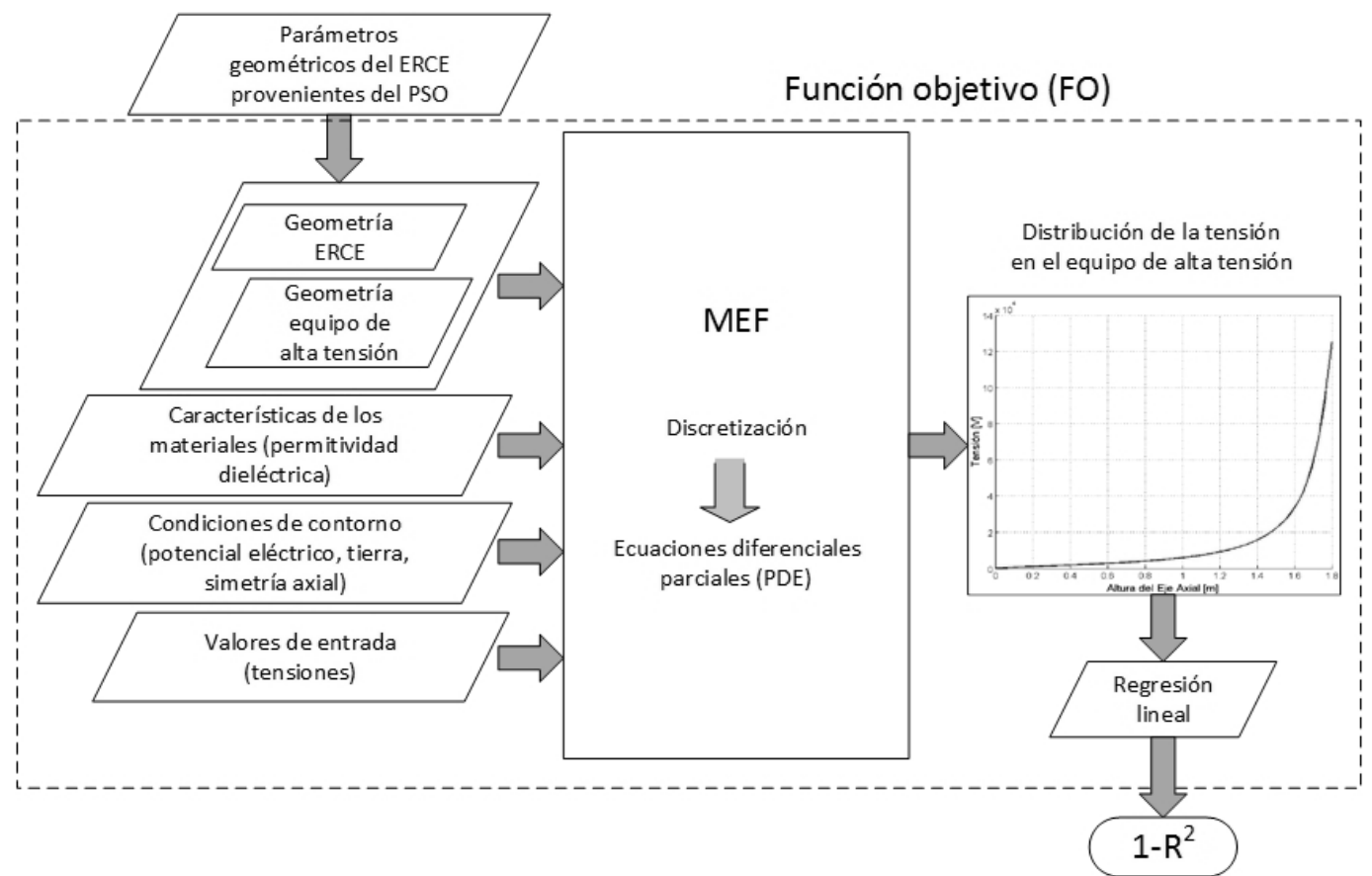

Fig. 2. Diagrama esquemático de la función objetivo. Fuente: Autores.

túa por medio del denominado coeficiente de determinación de regresión $\left(R^{2}\right)$, de acuerdo con (4).

$R^{2}=\frac{\left(n \sum x_{i} y_{i}-\sum x_{i} \sum y_{i}\right)^{2}}{\left(n \sum x_{i}^{2}-\left(\sum x_{i}\right)^{2}\right) *\left(n \sum y_{i}^{2}-\left(\sum y_{i}\right)^{2}\right)}$

Donde $n$ es el número de datos, $x_{i}$ son los valores de la posición sobre la trayectoria o contorno en el cual se evalúa la distribución de tensión y $y_{i}$ son los valores correspondientes a las tensiones a lo largo de esta trayectoria. Si $R^{2}$ es igual a 1 , los datos espaciales de la tensión sobre la trayectoria de interés son perfectamente lineales y se apartan de esta linealidad a medida que el coeficiente de determinación tiende a cero. Finalmente, como la optimización se puede tratar como un problema de minimización de la función objetivo, esta se define de acuerdo a (5), cuyo mínimo ideal es cero.

$F O=1-R^{2}$
El coeficiente de determinación (4) y la función (5), son dependientes de los parámetros geométricos del ERCE.

\section{EVALUACIÓN DE LA METOdología PROPUESTA}

Para la implementación y validación de la metodología de diseño de los ERCE, se empleó el software de elementos finitos COMSOL Multiphysics y el software MATLAB. En el MATLAB se desarrolló el proceso de optimización mediante PSO y la evaluación de la linealidad del voltaje y en la herramienta COMSOL se implementó y resolvió, mediante elementos finitos, el modelo electro-estático del equipo de alta tensión.

Para validar la funcionalidad de la metodología propuesta, se desarrolló un caso de estudio en el cual se realiza el diseño de dos tipos de electrodos relajadores de campo eléctrico de un divisor de tensión resistivo de $125 \mathrm{kV}$ empleado para la medición de ondas de impulsos de tensión tipo rayo. 


\subsection{Descripción y modelamiento MEF del divisor de tensión}

El divisor está conformado por una resistencia continua de alambre arrollada sobre un tubo de PVC, que a la vez está contenido dentro de otro tubo de PVC de mayor diámetro, el cual está lleno de aceite dieléctrico. La tensión de entrada del divisor está en la parte superior y en la parte inferior se supone una tensión igual a cero. En la 0 se presenta un corte del divisor y se muestran los materiales involucrados, también se presentan los dos tipos de ERCE, cuyos parámetros geométricos se optimizaron mediante la metodología propuesta en este trabajo.

Debido a la simetría del divisor, este se representó en el modelo de elementos finitos con una geometría $2 \mathrm{D}$ con simetría respecto del eje axial, es decir, para el análisis solo se requiere dibujar la mitad del divisor, lo cual reduce los tiempos de cómputo permitiendo mejoras en la precisión de los resultados. En la Tabla 1, se indican las permisividades de los materiales empleados en la simulación del divisor de tensión.

Las condiciones de frontera consideradas en el modelo fueron: simetría para el eje axial, tierra para el límite del espacio de simulación, de potencial para los elementos del divisor en tensión (0 V y 125 $\mathrm{kV}$ ) y de continuidad para las restantes.

La optimización de la geometría de los ERCE para los dos casos implementados se logró por medio de la parametrización de sus variables asociadas. Para el primer ERCE se parametrizó el diámetro de las placas paralelas denominada como la variable L en la 0 (b). Para el segundo ERCE se parametrizó la posición del ERCE tipo aro a través de las variables $\mathrm{P}$ y $\mathrm{Z}$ mostradas en la 0 (c). Los valores de los parámetros geométricos de estos ERCE son originados a partir del proceso de optimización PSO.

\subsection{Optimización de los ERCE del caso en estudio por medio de PSO}

Para la optimización de los electrodos relajadores de campo eléctrico se implementó el algoritmo PSO completo con factor de inercia. Para los coeficientes social y cognitivo $c_{1}$ y $c_{2}$ de 0 , se estableció un valor de 1,7 , este valor es el recomendado según los resultados del análisis de convergencia y parámetros de selección para el algoritmo de optimización PSO realizado por Trelea [17]. Para el factor de inercia $(w)$ que multiplica el valor precedente de la velocidad, se fijó un valor de 0,6 de acuerdo con lo sugerido en [18], y para la velocidad máxima se escogió un valor de 0,01.

Una vez establecidos los coeficientes, el factor de inercia y la velocidad máxima, se definió el número de variables del problema, una variable para el caso del ERCE de placas paralelas (L) y dos variables para el ERCE de tipo aro (P y Z). Después se limitó el área de búsqueda para los dos casos, para la variable L del ERCE de placas paralelas se asumió un rango de [0,07623 $\mathrm{m}-1 \mathrm{~m}]$, para el ERCE tipo aro se escogió un rango de $[0,25 \mathrm{~m}-1 \mathrm{~m}]$ para la variable $\mathrm{P}$ y de $[0 \mathrm{~m}-1,55 \mathrm{~m}]$ para la variable $\mathrm{Z}$.

En el caso de la variable $\mathrm{P}$ del ERCE tipo aro se escogió el límite inferior de tal manera que se reduzca la posibilidad de descarga disruptiva entre el aro del ERCE y el cuerpo del divisor, teniendo en cuenta las recomendaciones de la norma IEEE Std 4 [19]. 


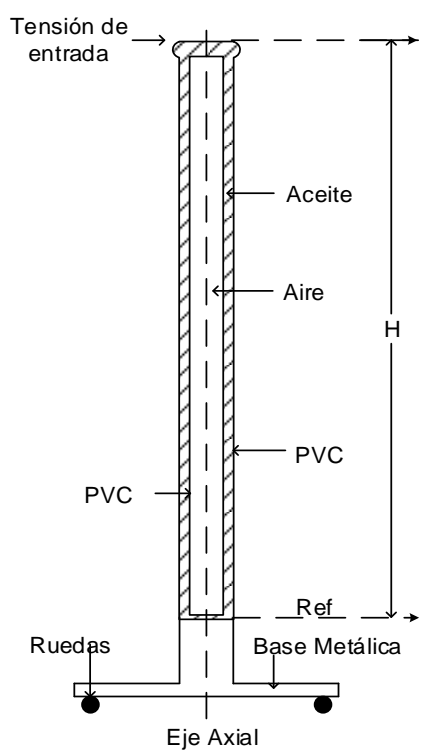

(a)

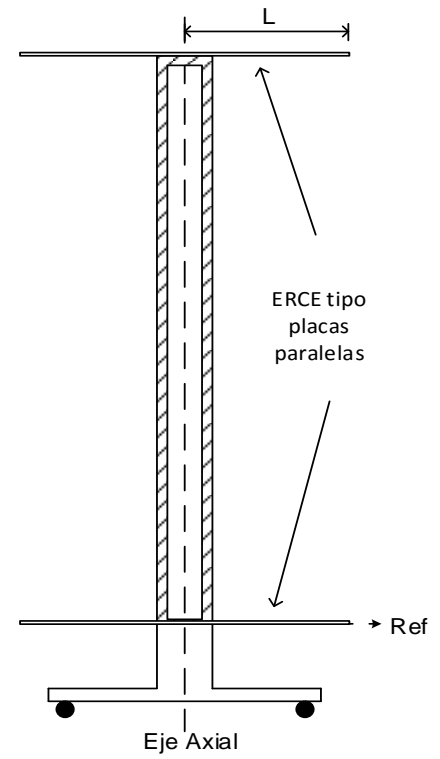

(b)

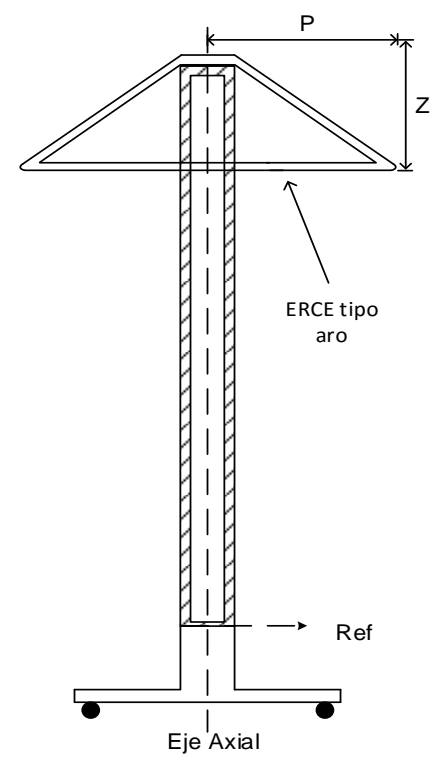

(c)

Fig. 3. Corte del divisor de tensión (a) sin ERCE, (b) con ERCE tipo placas paralelas, (c) con ERCE tipo aro. Fuente: Autores.

Tabla 1. Permisividades relativas de los elementos del divisor. Fuente: Cheng [16].

\begin{tabular}{llc}
\hline \multicolumn{1}{c}{ Pieza del divisor } & Material & $\begin{array}{c}\text { Permisividad } \\
\text { relativa }\left(\boldsymbol{\varepsilon}_{r}\right)\end{array}$ \\
\hline Medio circundante & Aire & 1 \\
Tubos interno y externo & PVC & 3,2 \\
Ruedas & Caucho & 2,5 \\
Cuerpo del divisor & Aceite & 2,3 \\
Base & Hierro & 1 \\
ERCE & Aluminio & 1 \\
\hline
\end{tabular}

Para facilitar la búsqueda del algoritmo PSO, se normalizaron todas las variables para que estas tomen valores en el rango de $[0$ - 1]. Posteriormente, para evaluar la función objetivo, se des-normalizan de acuerdo con (7).

$x=\hat{x}\left(x_{u b}-x_{l b}\right)+x_{l b}$

Donde, $x \in\left[x_{l b}, x_{u b}\right]$ es el valor con las dimensiones reales de los ERCE, $x_{u b}$ y $x_{l b}$ son el límite superior e inferior del pará- metro $x, \mathrm{y} \hat{x}$ representa el valor normalizado.

\subsection{Evaluación de los ERCE del caso en estudio por medio de PSO}

La 0 muestra la evolución del gbest durante uno de los procesos de optimización de la geometría de los ERCE. En estos dos casos en particular, el algoritmo PSO empleado se ajustó con diez partículas $(n=10)$ y un máximo de iteraciones de 50 $\left(k_{\max }=50\right)$, este último valor utilizado también como criterio de parada. 


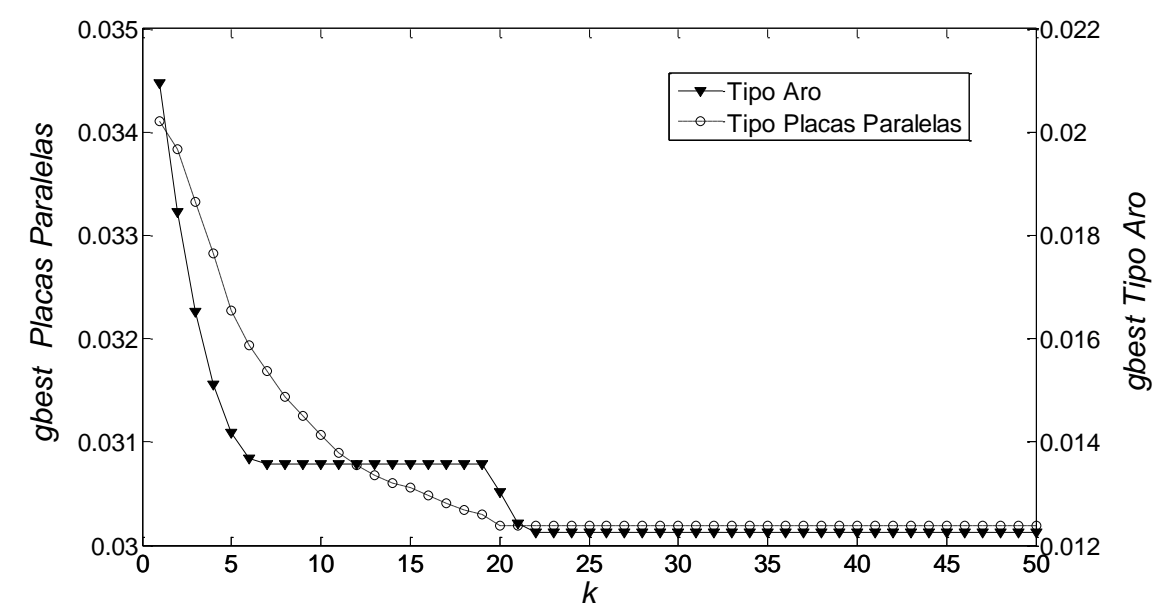

Fig. 4. Evolución del gbest con ERCE de placas paralelas y tipo aro, usando n=10 partículas y 50 iteraciones. Fuente: Autores.

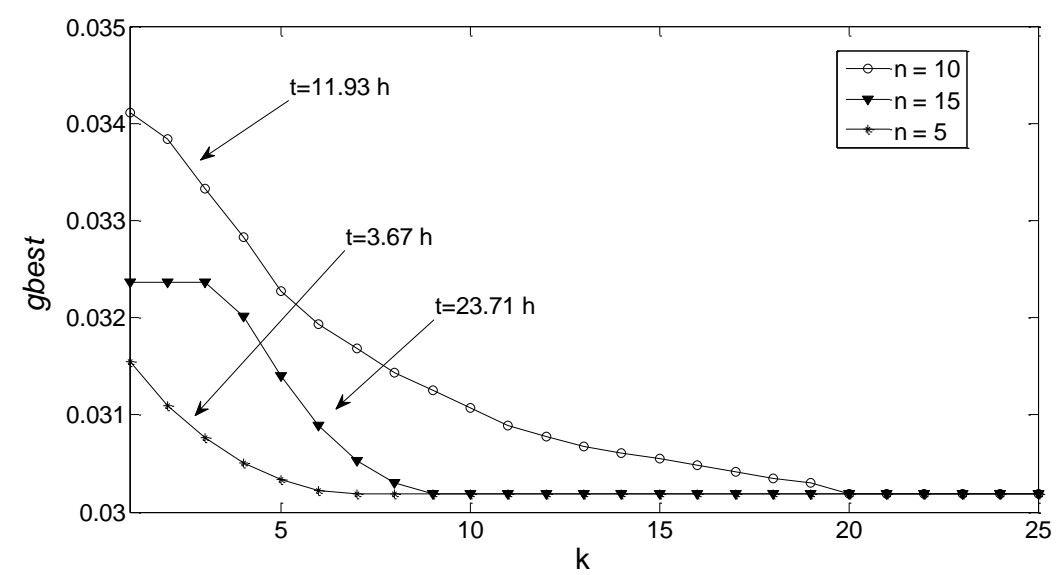

Fig. 5. Evolución del gbest con ERCE de placas paralelas, usando diferente número de partículas y un criterio de parada de 50 iteraciones. Fuente: Autores.

También se probó la metodología de optimización empleando diferente número de partículas. En la 0 se presenta la evolución del gbest del divisor con ERCE de placas paralelas, al variar el número de partículas $(n=5,10$ y 15). Se observa que el tener un mayor número de partículas actuando en el espacio de búsqueda, no implica necesariamente que la solución se alcance en un menor número de iteraciones, por el contrario, al aumentar el número de partículas el tiempo requerido para encontrar la mejor solución aumenta, porque en cada iteración es necesario que la función objetivo sea evaluada para cada una de las partículas.
Para reducir el tiempo requerido para la optimización del diseño se puede emplear el criterio de convergencia o parada tipo umbral (threshold). Por ejemplo, aplicando un criterio de convergencia o parada de gbest $<0,034$ y un número de partículas $n=10$, el tiempo requerido para la convergencia en el caso del divisor con ERCE de placas paralelas fue de $0,89 h$ (4 iteraciones) en vez de las $11,93 h$ que se requirieron cuando el criterio de parada fue el de agotar el número máximo de iteraciones $(k=50)$, sin embargo con este criterio de convergencia se obtiene una buena solución, pero no necesariamente la mejor posible. 
Así mismo, se comprobó la robustez de la metodología de optimización, tanto para el ERCE tipo placas paralelas como para el tipo aro, al repetir el proceso 30 veces, para cada uno de los valores de partículas mencionados $(n=5,10$ y 15$)$, encontrando diferencias inferiores al $5 \%$ entre los valores de los parámetros geométricos hallados para los ERCE.

Una vez obtenidos los parámetros geométricos para los dos tipos de ERCE, se procedió a simular el comportamiento del divisor de tensión en la herramienta de elementos finitos. La 0 presenta la comparación del potencial eléctrico y la 0 la comparación de los esfuerzos eléctricos a lo largo del eje axial del divisor resistivo, cuando se usan las dimensiones optimizadas para los ERCE, tipo placas paralelas $(L=1 \mathrm{~m})$ y tipo aro $(P=0,5453 \mathrm{~m}, Z=$ $0,5273 \mathrm{~m})$. Se aprecia cómo al emplear los ERCE ocurre una disminución significativa de los esfuerzos eléctricos y una mayor linealidad de la distribución de tensión a lo largo del eje axial del divisor, en especial cuando se usa el electrodo tipo placas paralelas.

\section{CONCLUSIONES}

Mediante el caso de estudio, se mostró la utilidad de la metodología propuesta para el diseño de los ERCE. La metodología propuesta aprovecha la fortaleza de la técnica numérica de los elementos finitos para el modelamiento de campos eléctricos y la capacidad de búsqueda metaheurística de la técnica de optimización por enjambre de partículas.

Se observó también que la metodología propuesta para el diseño de los ERCE, funciona adecuadamente inclusive con un número pequeño de partículas, aunque ello puede afectar el tiempo empleado en hallar la solución al diseño.

Uno de los aspectos principales para la utilización de la técnica de diseño optimizado, es que esta emplee tiempos aceptables para encontrar los parámetros del diseño. En ese sentido, hay cuatro aspectos en los cuales se puede trabajar a futuro para mejorar la metodología propuesta en este trabajo: el primero de ellos es mejorar el desempeño del modelo MEF, ya que allí se emplea la mayor proporción del tiempo requerido. El segundo tema consiste en emplear criterios de convergencia de tipo umbral, aunque para ello se requiere apelar a la experticia de los diseñadores. También se puede trabajar en determinar el número óptimo de partículas del enjambre. Finalmente, se pueden investigar variaciones al método clásico PSO empleado en este trabajo.

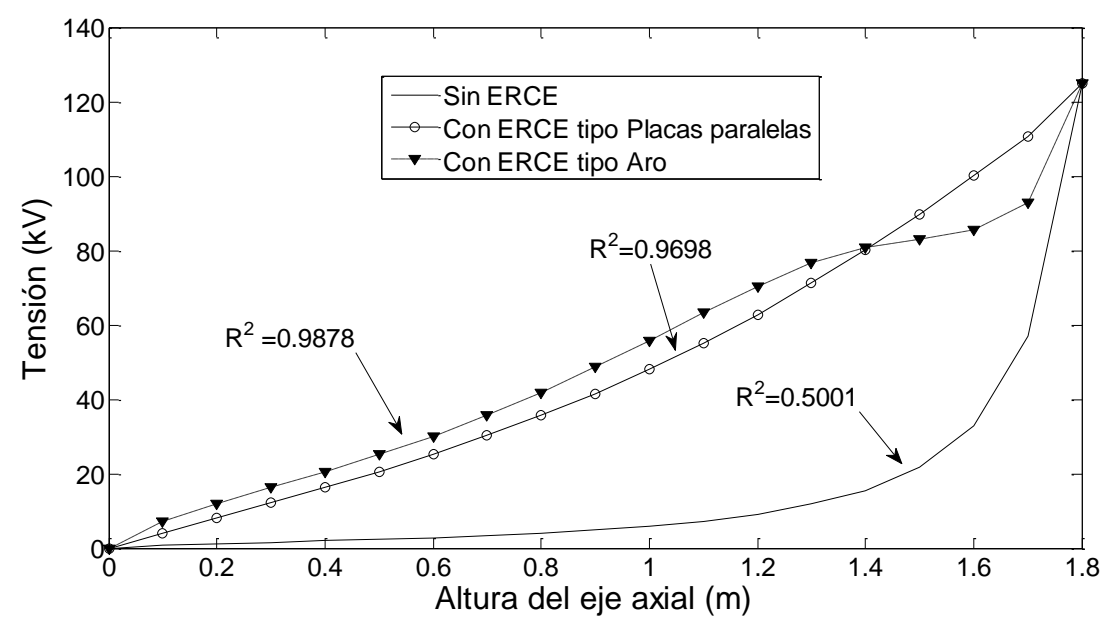

Fig. 6. Comparación del potencial eléctrico a lo largo del eje axial del divisor resistivo de 125 kV. Fuente: Autores. 


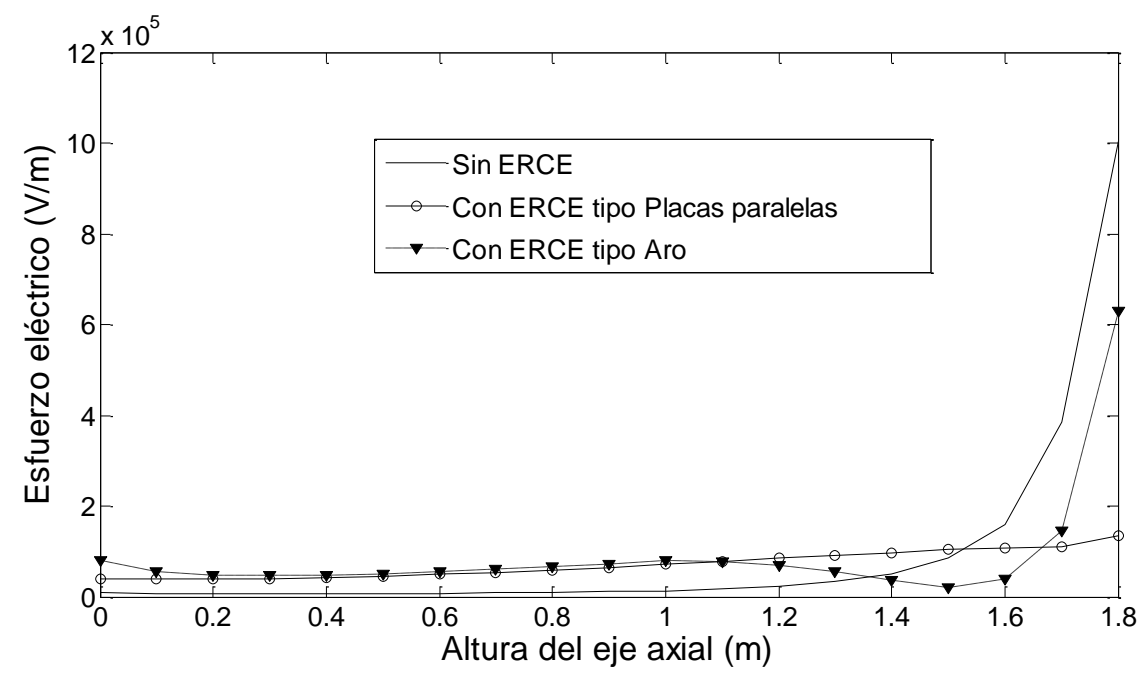

Fig. 7. Comparación de los esfuerzos eléctricos a lo largo del eje axial del divisor resistivo de 125 kV. Fuente: Autores.

\section{REFERENCIAS}

[1] D. F. García, E. M. Saens, T. A. Prado, and M. Martinez, "Metodology for Lightning Impulse Voltage Divisors Design," Latin America Transactions, IEEE (Revista IEEE America Latina), vol. 7, no. 1. pp. 71-77, 2009.

[2] N. Pattanadech, S. Potivetkul, and P. Yuttagowith, "Corona phenomena of various high voltage shielding types," in Power System Technology, 2006. PowerCon 2006. International Conference on, 2006, pp. 1-6.

[3] I. Feria and G. Gómez, "Diseño y manufactura de la base, el reductor y el mecanismo de torsión para el ensamblaje de una máquina educativa para prueba de torsión," México, 2011.

[4] C. Zhang, J. J. Kester, C. W. Daley, and S. J. Rigby, "Electric field analysis of high voltage apparatus using finite element method," in Electrical Insulation and Dielectric Phenomena (CEIDP), 2010 Annual Report Conference on, 2010, pp. 1-4.

[5] J. Du, Z. Peng, J. Li, S. Zhang, N. Li, and C. Fan, "Electric field calculation and grading ring optimization for $1000 \mathrm{kV}$ AC post porcelain insulator," in Solid Dielectrics (ICSD), 2013 IEEE International Conference on, 2013, pp. 198-201.

[6] S. Zhang, Z. Peng, L. Peng, and H. Wang, "Optimization of corona ring structure for UHV composite insulator using finite element method and PSO algorithm," in Solid Dielectrics (ICSD), 2013 IEEE International Conference on, 2013, pp. 210213.
[7] B. M'hamdi, M. Teguar, and A. Mekhaldi, "Optimal design of corona ring on $\mathrm{HV}$ composite insulator using PSO approach with dynamic population size," IEEE Trans. Dielectr. Electr. Insul., vol. 23, no. 2, pp. 1048-1057, 2016.

[8] C. Duarte and J. Quiroga, "Algoritmo PSO para identificación de parámetros en un motor DC System identification of a DC motor using PSO algorithm," Rev. Fac. Ing. Univ. Antioquia, no. 55, pp. 116-124, 2010.

[9] M. Heydarianasl and M. F. Rahmat, "Optimization of electrostatic sensor electrodes using particle swarm optimization technique," Int. J. Adv. Manuf. Technol., pp. 1-15, 2016.

[10] Z. Liu, J. Lu, and P. Zhu, "Lightweight design of automotive composite bumper system using modified particle swarm optimizer," Compos. Struct., vol. 140, pp. 630-643, 2016.

[11] R. C. Eberhart, J. Kennedy, and others, "A new optimizer using particle swarm theory," in Proceedings of the sixth international symposium on micro machine and human science, 1995, vol. 1, pp. 39-43.

[12] D. Merkle and C. Blum, "Swarm Intelligence: Introduction and Application." Springer Verlag Gmbh, 2008.

[13] R. Poli, "An analysis of publications on particle swarm optimization applications," Essex, UK Dep. Comput. Sci. Univ. Essex, 2007.

[14] M. Muñoz, J. López, and E. F. Caicedo, "Inteligencia de enjambres: sociedades para la solución de problemas (una revisión)," Ing. e Investig., vol. 28, no. 2, pp. 119-130, 2008.

[15] M. L. C. Cagnina, "Optimización mono y multiobjetivo a través de una heurística de 
Diseño de relajadores de campo eléctrico usando optimización por enjambre de partículas y el método de elementos finitos

inteligencia colectiva," Doctoral Thesis, Universidad Nacional de San Luis, 2010.

[16] D. K. Cheng, Fundamentos de electromagnetismo para ingeniería. Pearson Educación, 1998.

[17] I. C. Trelea, "The particle swarm optimization algorithm: convergence analysis and parameter selection," Inf. Process. Lett., vol. 85, no. 6, pp. 317-325, 2003.
[18] J. C. Bansal, P. K. Singh, M. Saraswat, A. Verma, S. S. Jadon, and A. Abraham, "Inertia weight strategies in particle swarm optimization," in Nature and Biologically Inspired Computing (NaBIC), 2011 Third World Congress on, 2011, pp. 633-640.

[19] "IEEE Standard for High-Voltage Testing Techniques," IEEE Std 4-2013 (Revision IEEE Std 4-1995), pp. 1-213, May 2013. 